\title{
Electronic consultation as an alternative to hospital referral for patients with chronic kidney disease: a novel application for networked electronic health records to improve the accessibility and efficiency of healthcare
}

\author{
John Stoves, ${ }^{1}$ John Connolly, ${ }^{2}$ Chee Kay Cheung, ${ }^{1}$ Angela Grange, ${ }^{3}$ Penny Rhodes, ${ }^{3}$ \\ Donal O'Donoghue, ${ }^{4}$ John Wright ${ }^{3}$
}

${ }^{1}$ Department of Renal Medicine, Bradford Teaching Hospitals NHS Foundation Trust, Bradford, UK

${ }^{2}$ Bradford and Airedale Primary Care Trust, Douglas Mill,

Bradford, UK

${ }^{3}$ Bradford Institute for Healthcare Research, Bradford Royal Infirmary, Bradford, UK ${ }^{4}$ Salford Royal NHS Foundation Trust, Hope Hospital, Stott Lane, Salford, UK

Correspondence to Dr J Stoves, Department of Renal Medicine, Bradford St Luke's Hospital, Little Horton Lane, Bradford BD5 ONA, UK; john.stoves@bradfordhospitals. nhs.uk

Accepted 20 February 2010 Published Online First 16 June 2010

\section{ABSTRACT}

Problem Chronic kidney disease is increasingly recognised in the UK, leading to a greater demand for specialist services. Traditional means of meeting this demand rely on GP referral of patients to see a nephrologist. Hospital assessment may be inconvenient for patients and inefficient for health services.

Setting 17 general practices and a secondary care nephrology service in Bradford, UK.

Design A before and after evaluation comparing nephrology referrals from implementation and nonimplementation practices following the introduction of electronic consultations (e-consultations) for chronic kidney disease.

Key measures for improvement The number, appropriateness and quality of new referrals (paper and electronic) from primary care, the timeliness of responses and the satisfaction of patients and health professionals with the new service.

Strategies for change Electronic sharing of primary care electronic health records with the nephrology service was introduced to implementation practices. Participating GPs attended education workshops and received paper and e-guidance about the new service. Effects of change There was a significant reduction in paper referrals from implementation practices. Econsultation provided nephrologists with access to more clinical information. GPs reported that the service was convenient, provided timely and helpful advice, and avoided outpatient referrals. Specialist recommendations were well followed, and GPs felt more confident about managing chronic kidney disease in the community. Lessons learnt $\mathrm{E}$-consultation promotes effective management of patients with mild-to-moderate chronic kidney disease in primary care, allowing specialist resources to be directed towards supporting patients with more complex needs. There is a potential role for econsultation in other chronic disease specialties.

\section{CONTEXT}

Primary care teams in the UK have recently assumed greater responsibility for the management of chronic kidney disease in recognition of the need to identify the condition at an early stage and then intervene to slow renal disease progression and reduce cardiovascular morbidity and mortality. ${ }^{1}{ }^{2}$
A number of recent policy initiatives have helped to facilitate this transition. ${ }^{3-6}$ The Quality and Outcomes Framework, first introduced in 2004, is a reward and incentive programme for UK general practices that details performance across a range of clinical care standards, and in 2006 it was extended to include chronic kidney disease. In 2004, the UK Department of Health published the National Service Framework for Renal Services, in which it is recommended that the referral of patients with progressive chronic kidney disease from primary care to specialist renal services should occur at an appropriate stage to optimise treatment outcomes. Achievement of this clinical standard requires both the provision of clear management guidance for GPs and a consistently high quality of communication between primary and secondary care. ${ }^{56}$

The Bradford and Airedale Primary Care Trust serves a population of approximately 0.6 million people in a mixed urban and rural district in the North of England. It is estimated that more than $8 \%$ of adults in the region have chronic kidney disease stages $3-5$, with case detection improving nationally. ${ }^{\text {? }}$ The rising prevalence of recognised chronic kidney disease prompted a multidisciplinary review of local renal service provision, which led to the initiation of a programme of work with GPs to strengthen communication at the interface between primary and secondary care using electronic methods. ${ }^{8}$ Over $90 \%$ of primary care practices in the Bradford and Airedale Primary Care Trust use a centralised IT system (SystmOne) that allows the sharing of detailed electronic health records by groups of healthcare professionals in various care settings. ${ }^{9}$

\section{OUTLINE OF PROBLEM}

Primary care records are generally not accessible to secondary care staff, and consequently telephone calls and referral letters are the principal means of communication between community and hospital teams. These processes are often inadequate. Some patients who are referred to the hospital renal clinic can be managed more appropriately in primary care (with ready access to expertise in secondary care), allowing specialist resources to be directed towards patients with more complex needs. The availability to nephrologists of the clinical information 
contained in primary care records is likely to improve communication and hence support the management of patients with relatively mild and non-progressive chronic kidney disease in the community. The potential benefits of using electronic health records to enhance the quality, safety and efficiency of healthcare have been recognised. ${ }^{10}$ Reported studies have focused mainly on 'stand alone' systems such as those used by individual general practices. ${ }^{11-14}$ In this study, we have explored electronic consultation (e-consultation) using networked electronic health records to support the ongoing management of patients with mild-to-moderate chronic kidney disease in primary care and so avoid the need for initial assessment in a hospital clinic.

\section{STRATEGY FOR CHANGE}

We developed a chronic kidney disease e-consultation service in SystmOne. The service allows GPs to send electronic referrals and share patient electronic health records with a renal specialist after first obtaining verbal patient consent. GPs use criteria agreed in local guidelines to 'request advice' or 'question the need for hospital clinic review. ${ }^{15}$ The renal specialist is able to open the electronic health record and view important clinical details such as patient comorbidities, medication history, lifestyle factors, previous communications from other specialists, reports of previous imaging and a chronological display of selected numerical data (blood pressure, estimated glomerular filtration rate, blood biochemistry and urinalysis). A decision is then made as to whether a patient should be referred to clinic, undergo tests or interventions in the primary care setting, or continue to be monitored and treated by the primary care team. Physician time is saved by using preset 'auto-consultation' responses. Responses are saved in the patient's electronic health record and also sent as tasks to alert the referring primary care team.

A single practice pilot of e-consultation indicated potential benefits, with better coordination of patient management and avoidance of clinic referrals. We therefore introduced e-consultation to 17 volunteer implementation practices in July 2007, supported by two nephrologists. Participating GPs attended education events and received paper and electronic guidance about the new service. It was explained that the service should be used to obtain advice for specific queries and to request virtual review of patients with an indication for hospital clinic referral that was 'borderline' according to local criteria. ${ }^{15}$

\section{PROCESS OF GATHERING INFORMATION}

We evaluated the number, appropriateness and quality of all paper and electronic chronic kidney disease referrals made by GPs to the Bradford Renal Unit, the timeliness of responses made by the renal specialists and the subsequent actions taken. Professional and patient views were also assessed.

\section{Quantitative data}

We analysed paper referrals from 17 implementation and 68 nonimplementation practices (all other practices served by the Bradford Renal Unit) over a run-in period prior to the introduction of the e-consultation service (January 2005 to August 2007) to provide baseline data. Data for all new paper and electronic referrals in the 12 months following the introduction of the service (September 2007 to September 2008) were collected prospectively using a structured proforma. The participating nephrologists subjectively categorised referrals according to whether the GP was seeking specific advice or questioning the need for review in the hospital clinic.

\section{Qualitative data}

Nephrologists $(n=2)$ and GPs who had used the service $(n=35)$ were offered a choice of telephone interview or email questionnaire. Content validity of interview schedules and questionnaires was assured through clinician review. Thematic analysis of the qualitative data was performed manually to identify key issues and themes using the constant comparative method. ${ }^{16}$

\section{ANALYSIS AND INTERPRETATION}

Figure 1 summarises the pattern and quality of all primary care paper $(n=376)$ and electronic $(n=68)$ new chronic kidney disease referrals for 12 months following the introduction of e-consultation. The mean age of patients referred electronically was greater than patients referred by letter ( 72.8 years vs 68 years, $\mathrm{p}<0.01)$. There was no significant difference in reported comorbidities such as diabetes mellitus, hypertension, ischaemic heart disease, peripheral vascular disease and cerebrovascular disease. The mean (SE) interval between the GP sending an e-consultation referral and the renal specialist submitting an electronic response was 7 (0.8) days. This contrasted with a mean wait of 55.1 (1.6) days between the GP sending a paper referral and the patient attending a hospital clinic. The mean (SE) time to perform an e-consultation was 15.5 (0.4) min. E-consultations provided the renal specialist with access to more
Figure 1 Flow chart for patients with chronic kidney disease who were referred by GPs to Bradford renal services between September 2007 and September 2008. Eleven out of $68(16 \%)$ e-consultations were finally referred to the clinic, compared with 376 out of the $398(94 \%)$ paper referrals.

*'Appropriate' and 'borderline' were defined according to local chronic kidney disease referral guidelines. ${ }^{15}$

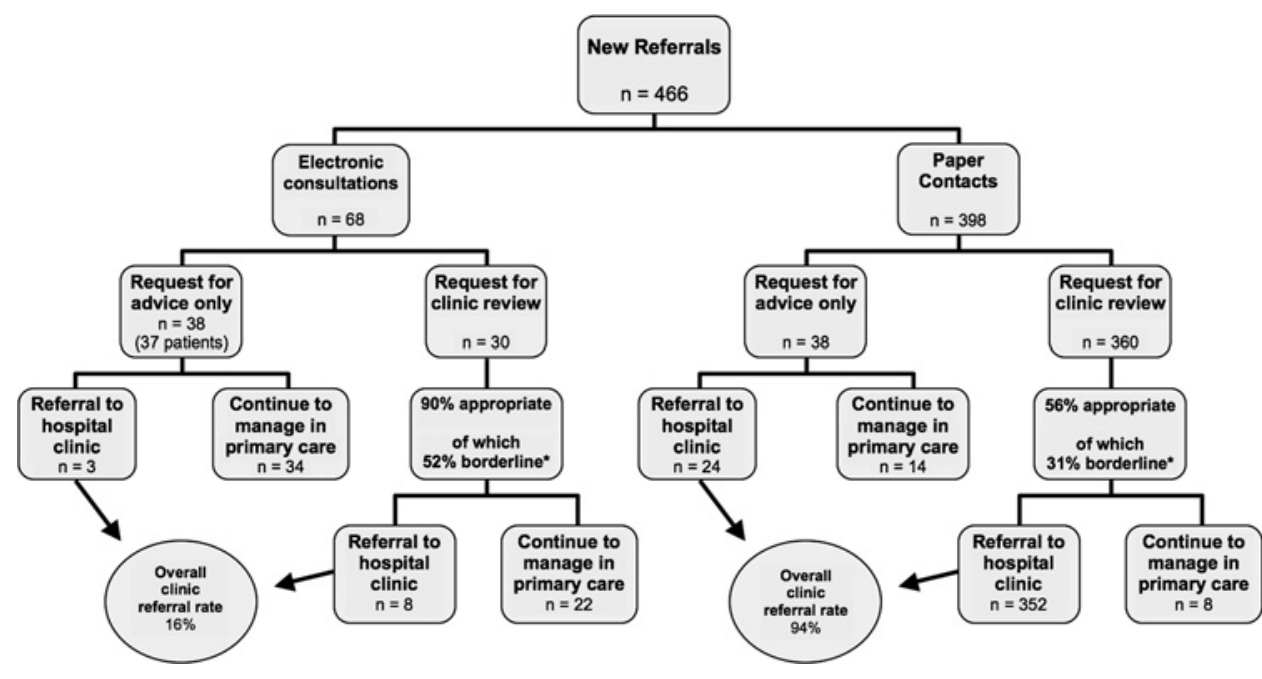


clinical information; for example, there were 14 (1.3) previous serum creatinine measurements per referral compared with only two (0.1) measurements in paper referrals, and urinalysis data were available in $76.7 \%$ of e-consultations compared with $30.8 \%$ of paper referrals.

When GPs were requesting clinic review by letter, only $56 \%$ of referrals were appropriate according to local criteria $(71 \%$ and $52 \%$ for implementation and non-implementation practices respectively), but $98 \%$ of these were accepted for hospital clinic review. This may have been due to insufficient information in some referral letters and a general reluctance to cancel appointments that had been prebooked by the GP or the patient. By contrast, $90 \%$ of e-consultations that questioned the need for clinic review were appropriate, and clinic assessment was recommended in only $27 \%$ of cases (figure 1 ). GPs were asked to arrange additional investigations in advance of the clinic appointment where appropriate.

The electronic health records of patients referred via the e-consultation service were reviewed several months after the study period had ended. For those in whom hospital clinic referral had been deemed unnecessary $(n=56)$, adherence to electronic advice was assessed. Recommendations made by the nephrologist for additional tests, treatment changes and monitoring schedules were adhered to in $82 \%, 97 \%$ and $89 \%$ of cases respectively. Reassuringly, renal function remained stable in this subgroup, with a small increase in estimated GFR of 3.6 (1.1) $\mathrm{ml} / \mathrm{min} / 1.73 \mathrm{~m}^{2}$ after a mean follow-up period of 8.8 (0.7) months in primary care.

\section{EFFECTS OF CHANGE}

GP interviews and questionnaire responses to structured questionnaires (response rate 46\%, 16/35) elicited similar themes. E-consultation was regarded as a convenient service that provided timely and helpful advice, and avoided unnecessary referral to the hospital clinic. GPs recognised that e-consultation presented an educational opportunity that increased their confidence in managing chronic kidney disease in the community. Less positive comments referred to difficulties using the system (problems which have been rectified) and some concerns over patient privacy, although patients were generally willing to consent to the viewing of their electronic health record by a renal specialist. Opportunistic interviews with GPs and patients revealed strong patient support for e-consultation on the grounds of service efficiency and avoidance of unnecessary hospital visits.

For the nephrologist, e-consultation permitted a detailed and efficient review of a patient's primary care electronic health record, facilitating prompt and informed decision-making. Patients in need of renal outpatient clinic assessment were readily identified, and others benefited from the provision of timely advice. Avoidance of unnecessary hospital clinic visits was seen as an effective way of releasing resources in the specialist unit for those patients who need them most, as well as saving on transport and other environmental costs.

Paper and electronic referral activity for implementation and non-implementation practices is summarised in figure 2. GPs from the implementation practices were briefed about the e-consultation service during the quarter preceding its introduction. The fall in paper referrals for this quarter may be explained by anticipation of the new service. When data from this quarter were considered as part of the postimplementation period in our referral analysis, we found that the ratio of paper referrals made post-versus preintroduction of the e-consultation service was 0.54 (95\% CI 0.42 to 0.71 ) for the implementation practices, compared with 0.98 (95\% CI 0.86 to 1.12$)$ for the

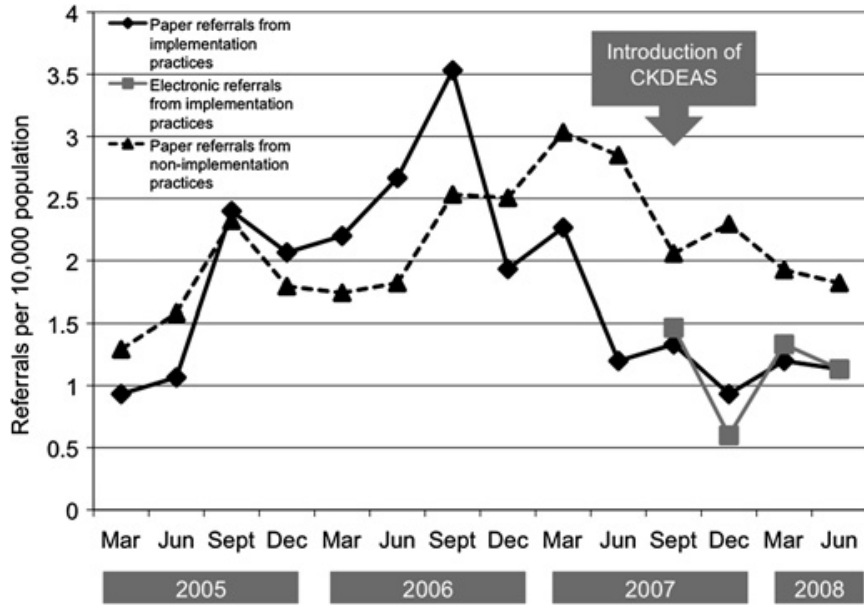

Figure 2 Quarterly rates of GP referral to renal services of patients with chronic kidney disease.

non-implementation practices. When data from the same quarter were considered as part of the preimplementation period, similar results were obtained (rate ratios of 0.57 (95\% CI 0.44 to 0.74 ) and 0.94 (95\% CI 0.83 to 1.08 ), respectively).

The total referral rate (counting both paper and electronic referrals) was marginally higher for the implementation practices during the study period. Approximately half of e-consultations were requests for simple advice rather than an assessment of the need for clinic review of patients with 'borderline' chronic kidney disease (figure 1). Such requests may diminish over time as GPs become more familiar with chronic kidney disease management. There may also have been an initial 'washout' period during which GPs requested e-consultations for patients who were already known to have 'borderline' chronic kidney disease.

\section{LESSONS LEARNT AND NEXT STEPS}

IT interventions are being introduced rapidly and on a large scale across health services. Randomised controlled trial evaluation of their effect on service provision would be ideal, but this is rarely possible. Our pragmatic evaluation attempts to provide reliable evidence of the impact of a novel application for networked electronic health records. We have found that e-consultation is a feasible way of assessing patients with chronic kidney disease of mild-to-moderate severity. The process is efficient and facilitates informed decision-making. Professionals and patients have welcomed the service, and there has been recognition of the associated environmental benefits. ${ }^{17} \mathrm{We}$ intend to make e-consultation available to all eligible practices in Bradford and Airedale PCT. It is not yet clear as to whether the service will displace outpatient clinic activity or create additional work for nephrologists because of previous unmet need. Although there may be a continuing growth in chronic kidney case ascertainment driven by the renal Quality and Outcomes Framework, these data indicate that paper referral rates in Bradford are likely to diminish when the e-consultation service is made available to all practices. Uptake of the service may of course be different in practices that were not part of the initial evaluation and in healthcare settings outside the Bradford region. The summative effect of e-consultation activity on the workload of renal specialists is therefore not likely to be excessive, although in some centres the prevailing opportunity cost of seeing unnecessary referrals may be such that any new clinic capacity that arises from a reduction in such referrals will prompt more 
frequent review of patients with complex renal disease. Total activity will need to be monitored to ensure that the introduction of an e-consultation service does not create financial disincentives for commissioners or service providers.

There is interest in adopting this innovation in other UK renal services and also other chronic disease specialties. A recent paper describes a similar approach in the USA based on unsolicited e-consultations provided by nephrologists to generalists to ensure timely referral of patients who may require renal replacement therapy. ${ }^{18}$ We are also looking to create additional 'spokes' for our electronic renal hub to support medicines reconciliation and care pathways that are shared with GPs (such as anaemia management, predialysis care, palliative care and renal transplant care). This may reduce administration time and improve patient safety through more efficient communication.

Secondary care IT infrastructures will need to embrace primary care systems if e-consultation services are to be adopted more widely in the NHS. Regional initiatives supported by Yorkshire and the Humber SHA may lead to formal commissioning of such services in the future.

Acknowledgements We are indebted to L Speller, J Parry, C Durkin, R Whitaker, $K$ Farrar and the PACE team, for helping to set up, advertise and support the econsultation service; R Roberts, for performing e-consultations and recording data; $\mathrm{L}$ Thorp, for recording data; A Wong, for maintaining a database and providing data reports; L Fairley, for assisting with the quantitative analysis; J Keen, for commentary on the progress of the study; and R Pope, for sharing his experience of using electronic health records in diabetes care.

Funding NIHR Quality and Safety Programme.

Competing interests None.

Patient consent Obtained.

Ethics approval Ethics approval was provided by the Bradford Research Ethics Committee.

Contributors JS codeveloped a prototype of the e-consultation service, colaunched the service for implementation practices, set up the study, collected data, performed e-consultations, prepared the first draft and coordinated the writing of the paper. JC codeveloped a prototype of the e-consultation service, colaunched the service for implementation practices and helped to write the paper. CKC collected data, performed data analysis and helped to write the paper. AG helped to set up the study, collected qualitative data and reviewed the manuscript. PR collected and analysed qualitative data and helped to write the paper. DO'D supported the study and helped to write the paper. JW helped to set up the study, chaired review meetings, performed data analysis and helped to write the paper. JS is the guarantor of the study.
Provenance and peer review Not commissioned; externally peer reviewed.

\section{REFERENCES}

1. Feehally J, Griffith $\mathrm{KE}$, Lamb EJ, et al. Early detection of chronic kidney disease. BMJ 2008;337:a1618.

2. Go AS, Chertow GM, Fan D, et al. Chronic kidney disease and the risks of death, cardiovascular events, and hospitalization. $N$ Engl J Med 2004;351:1296-305

3. NHS Employers. Quality and outcomes framework guidance for GMS contract 2009/10. p101-6. http://www.nhsemployers.org/Aboutus/Publications/Documents/ QOF Guidance 2009 final.pdf (accessed 14 Sep 2009)

4. Richards N, Harris K, Whitfield M, et al. Primary care-based disease management of chronic kidney disease (CKD), based on estimated glomerular filtration rate (eGFR) reporting, improves patient outcomes. Nephrol Dial Transplant 2008:23:549-55.

5. Department of Health. National service framework for renal services - part two: chronic kidney disease, acute renal failure and end of life care. London: $\mathrm{DoH}$, 2005:p1-30.

6. Joint Specialty Committee on Renal Medicine of the Royal College of Physicians of London and the Renal Association. Chronic kidney disease in adults: UK guidelines for identification, management and referral. London: RCP, 2006

7. Stevens PE, O'Donoghue DJ, de Lusignan S, et al. Chronic kidney disease management in the United Kingdom: NEOERICA project results. Kidney Int 2007:72:92-99.

8. Tillett E, Mumtaz R, Mercer K, et al. A multidisciplinary approach to vascular access management: the Bradford Early Vascular Access (BEVA) project. Renal Association Annual Meeting 2007; P148.2010.

9. Connecting for Health [internet]. NHS. http://www.connectingforhealth.nhs.uk/ systemsandservices/gpsupport/gpsoc (accessed 14 Sep 2009).

10. Delpierre C, Cuzin L, Fillaux J, et al. A systematic review of computer-based patient record systems and quality of care: more randomised clinical trials or a broader approach? Int J Qual Health Care 2004:16:407-16.

11. Garg AX, Adhikari NK, McDonald H, et al. Effects of computerized clinical decision support systems on practitioner performance and patient outcomes: a systematic review. JAMA 2005:293:1223-38.

12. Poissant $\mathbf{L}$, Pereira J, Tamblyn $\mathrm{R}$, et al. The impact of electronic health records on time efficiency of physicians and nurses: a systematic review. J Am Med Inform Assoc 2005:12:505-16.

13. Roland M, McDonald ER, Sibbald BS, et al. Outpatient services and primary care. A scoping review of research into strategies for improving outpatient effectiveness and efficiency. Manchester: National Primary Care Research and Development Centre, 2006.

14. Smith SM, Allwright S, O'Dowd T. Effectiveness of shared care across the interface between primary and specialty care in chronic disease management. Cochrane Database Syst Rev 2007; (3): CD004910.

15. PACE. Algorithm to guide the management of adult patients with chronic kidney disease (CKD) Stages 1-5. Bradford and Airedale Teaching PCT. 2006. http://www. learnonline.nhs.uk/pace (accessed 14 Sep 2009).

16. Glaser BG, Strauss AL. The discovery of grounded theory: strategies for qualitative research. Chicago: Aldine Publishing Company, 1967.

17. Langton K, Mortimer F. The future of 'green' nephrology in the UK. Br J Ren Med 2009;14:8-9

18. Lee BJ, Forbes $K$. The role of specialists in managing the health of populations with chronic illness: the example of chronic kidney disease. BMJ 2009:339:b2395. 